\title{
Fault Diagnosis and Prognosis Based on Deep Belief Network and Particle Filtering
}

\author{
Guangxing $\mathrm{Niu}^{1}$, Shijie Tang ${ }^{2}$, Zhichao $\mathrm{Liu}^{3}$, Guangquan Zhao ${ }^{4}$, and Bin Zhang ${ }^{5}$, \\ 1,2,3,5 University of South Carolina, Columbia, South Carolina, 29169, USA \\ gniu@email.sc.edu \\ shijie@email.sc.edu \\ zhichao@email.sc.edu \\ zhangbin@cec.sc.edu \\ ${ }^{4}$ Harbin Institute of Technology, Harbin, Heilongjiang, 150006, China \\ hit53zhao@sina.com
}

\begin{abstract}
Fault diagnosis and prognosis (FDP) plays more and more important role in industries FDP aims to estimate current fault condition and then predict the remaining useful life (RUL). Based on the estimation of health state and RUL, essential decisions on maintenance, control, and planning can be conducted optimally in terms of economy, efficiency, and availability. With the increase of system complexity, it becomes more and more difficult to model the fault dynamics, especially for multiple interacting fault modes and for fault modes that are affected by many internal and external factors. With the development of machine learning and big data, deep learning algorithms become important tools in FDP due to their excellent performance in data processing, information extraction, and automatic modeling. In the past a few years, deep learning algorithms demonstrate outstanding performance in feature extraction and learning fault dynamics. As emerging techniques, their powerful learning capabilities attract more and more attentions and have been extended to various applications. This work presents a novel diagnosis and prognosis methodology which combined deep belief networks (DBNs) and Bayesian estimation. In the proposed work, the DBNs are trained offline using available historical data. The fault dynamic model is then represented by the trained DBNs and modeling uncertainty is described by noise. The integration of DBNs with particle filtering is then developed to provide an estimation of the current fault state and predict the remaining useful life, which is very suitable and efficient for most nonlinear fault models. Experimental
\end{abstract}

\footnotetext{
Guangxing Niu et al. This is an open-access article distributed under the terms of the Creative Commons Attribution 3.0 United States License, which permits unrestricted use, distribution, and reproduction in any medium, provided the original author and source are credited.
}

studies of lithium-ion batteries are presented to verify the effectiveness of the proposed solution.

\section{INTRODUCTION}

Engineering system are usually exposed to many stresses that can affect system reliability, safety, and mission effectiveness. Prognostic Health Management (PHM) is critical to these systems and fault diagnosis and prognosis are fundamental enabling techniques of PHM. Diagnosis is to detect fault and estimate the fault state. The fault severity is given by the discrepancy between a baseline (no-fault) probability density function (pdf) and a real-time estimation pdf of fault state. Prognosis is to predict the time to failure (TTF) or RUL. Here, RUL is the time from current time to failure time, which is defined as the time when the fault state reaches a predefined failure threshold. Based on the information of fault state and RUL obtained by FDP, maintenance activities can be scheduled and control can be reconfigured optimally and efficiently to lower operation and maintenance cost and increase the safety and availability of the system.

In the past a few years, numerous efforts on diagnosis and prognosis were developed (Z. Zhang, Wang, \& Wang, 2013; Orchard \& Vachtsevanos, 2009; Yan, Zhang, Wang, Dou, \& Wang, 2016; R. Zhao et al., 2018; G. Zhao et al., 2018; Yan et al., 2018; Hu et al., 2018). In general, these approaches can be classified into physics model-based approaches and datadriven based ones (Kan, Tan, \& Mathew, 2015; Jardine, Lin, $\&$ Banjevic, 2006). For physics model-based approaches, extensive knowledge about fault mechanism are often needed to build a fault degradation model, which is difficult and, in most cases, ad-hoc, which limits the application of model-based approaches for complicated systems. Data-driven based approaches, on the contrary, mainly rely on historical monitor- 
ing data to extract features and describe fault dynamic behaviors. Although these approaches do not require the prior knowledge about fault mechanism, they often require statically sufficient data. Many of the data-driven based methods have been developed ranging from statistical model, machine learning, to artificial intelligent methods. These approaches are often used for analyzing the available historical and conditional data in different ways for fault dynamics modeling, anomaly detection, diagnosis, and prognosis (Y. Wang, Peng, Zi, Jin, \& Tsui, 2016; Biswas, Srivastava, \& Whitehead, 2015; Mosallam, Medjaher, \& Zerhouni, 2016). However, these existing methods have some limitations that hinder their applications in complicated systems: (1) most features are manually extracted and selected, which require complex signal processing and extensive expert involvement; (2) feature extraction and selection for a fault model is ad-hoc and cannot be extended to other fault models; and (3) they have shallow architectures, which limit the capacity to learn the complex non-linear relationships in complex systems.

In recent years, deep learning related algorithms, such as Deep Belief Networks, Deep Neural Networks (DNNs) and Convolutional Neural Networks (CNNs), have drawn more and more attention due to their excellent achievements in image recognition and speech processing (Titos, Bueno, García, \& Benítez, 2018; Oquab, Bottou, Laptev, \& Sivic, 2014; Kang \& Meng, 2014). DBNs show superior abilities in feature extraction and have been used for FDP of many systems (Chen \& Li, 2017; Tamilselvan \& Wang, 2013; G. Zhao et al., n.d.). It is certain that the powerful feature extraction and learning abilities of DBNs can be explored and extended to many other applications. With this motivation, this paper presents a novel fault diagnosis and prognosis methodology, which integrates DBNs in particle filtering by combining the advantages of both methods to improve the performance in FDP. The lithium-ion battery capacity data are employed to validate the proposed approach. The results of the case study demonstrate the efficiency of the proposed solution.

This paper is organized as follows: Section 2 briefly describes the theoretical background and basic concepts that are related to this work. Section 3 presents the framework of the propose method, which is followed by details on the particle filtering based diagnosis and prognosis and the integration of DBN in particle filter. Section 4 presents the results and analysis of the proposed algorithms by application to the case studies of Lithium-ion batteries. Finally, Section 5 provides concluding remarks and some future research directions.

\section{THEORETICAL BACKGROUND}

In the proposed approach, DBNs are integrated into the framework of particle filtering based diagnosis and prognosis. The theory of DBNs, including its structure and training rules, will be discussed in this section together with the in- ference of Bayesian estimation theory and its approximation method, namely particle filtering.

\subsection{Deep Belief Networks}

DBN has shown demonstrated successes in feature extraction and data dimension reduction (X. Wang, Li, Rui, Zhu, \& Fei, 2015; G. Zhao et al., n.d.). It has become popular with its capabilities in capturing the representative information from raw time series data. DBN has a multi-layer feedforward structure of Restricted Boltzmann Machines (RBMs) (Van Tung Tran \& Ball, 2014), as shown in Fig. 1. With this multi-layer structure, DBN can extract fault feature layer by layer. The extracted features are used as input of the classifier for fault detection.

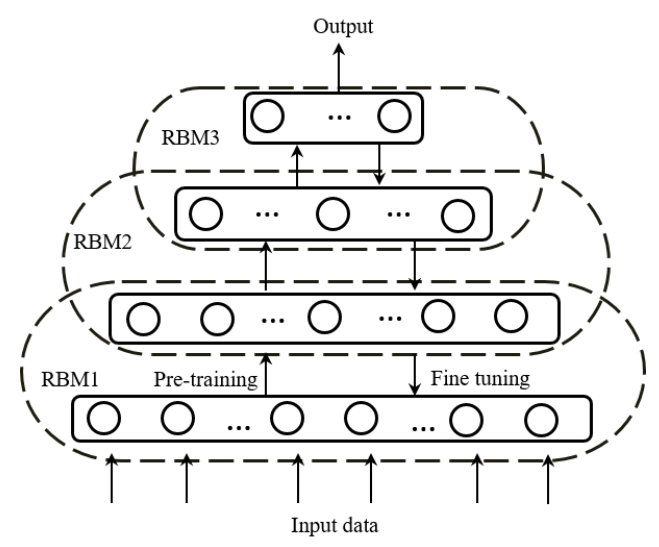

Figure 1. The structure of a 3-layer DNB

RBM is a special probabilistic model of Boltzmann machine, which consists of a visible input vector $v$ and a hidden vector $h$, connected by weighting factors, as shown in Fig. 2.

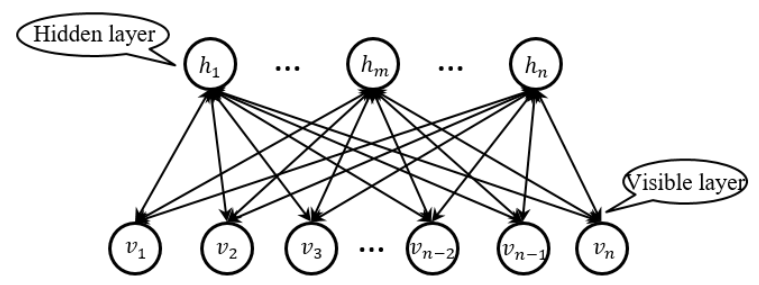

Figure 2. Structure of restricted Boltzman machine

The joint configuration $(v, h)$ can be given by the energy function (1)

$$
E(v, h)=-\sum_{j=1}^{m} a_{j} v_{j}-\sum_{i=1}^{n} b_{i} h_{j}-\sum_{i=1}^{n} \sum_{j=1}^{m} v_{j} w_{i j} h_{i}
$$

where $v_{i}$ and $a_{i}$ are the binary states and bias of the $i$-th element of the visible vector, $h_{j}$ and $b_{j}$ are the binary states and bias of the $j$-th element of the hidden vector, $w_{i j}$ is the 
weight of the connection between the visible layer and the hidden layer. The energy is used to assign a probability value to each state in visible and hidden units. The joint distribution is defined as

$$
p(v, h)=\frac{1}{Z} e^{-E(v, h)}
$$

where $Z$ is a partition function, which can be described as

$$
Z=\sum_{v, h} e^{-E(v, h)}
$$

As mentioned earlier, the connections just exist between the visible layer and the hidden layer. The neurons in the same layer are independent with each other. The conditional probabilities of the hidden layer and the visible units are given as

$$
\begin{aligned}
& p\left(h_{i}=1 \mid v\right)=\frac{1}{1+e^{-b_{i}-\sum_{i=1}^{m} v_{i} w_{i j}}} \\
& p\left(v_{i}=1 \mid h\right)=\frac{1}{1+e^{-a_{i}-\sum_{j=1}^{m} h_{j} w_{i j}}}
\end{aligned}
$$

The learning process of DBN can be divided into two stages: pre-training and fine-tuning (C. Zhang, Lim, Qin, \& Tan, 2017). In the pre-training process, the RBMs are trained layer by layer with an unsupervised manner. The forward pre-training process can be regarded as a construction and reconstruction process using Eq. (1). After all the RBMs in the DBN are pre-trained, the fine-tuning step will be applied to DBN using back propagation algorithm (Bengio, Lamblin, Popovici, \& Larochelle, 2007). In this fine-tuning process, the weights and biases of every layer are adjusted continuously until the errors satisfy the defined values. The trained DBN model is obtained after the fine-tuning step and can be used in describing the fault dynamics.

\subsection{Particle Filtering}

The fault processes of most engineering systems can be described by dynamic models, which include a process model and a measurement model. The nonlinear process model can be defined as:

$$
x_{k}=f_{k}\left(x_{k-1}, \omega_{k}\right)
$$

where $x_{k}$ is the fault state at time instant $k, \omega_{k}$ is the process noise, and $f(\cdot)$ is a nonlinear function representing the state transition. The measurement is given by assuming that the measurements $y$ are conditionally independent with state $x$. The model can be described by Eq. (7):

$$
y_{k}=h_{k}\left(x_{k}, v_{k}\right)
$$

where $y_{k}$ is the observed value at time instant $k, v_{k}$ is the observation noise.

In the Bayesian estimation framework, our objective is to use the dynamic fault model and historical observations to estimate the current fault state of the system by using the Bayesian theorem. Therefore, it is of interest to estimate the posterior distribution $p\left(x_{0: k} \mid y_{1: k}\right)$. The Bayesian estimation method usually involves two steps, i.e., prediction and filtering. The prediction step calculates the prior probability density function (pdf) by using the fault dynamic model. It is calculated as:

$$
p\left(x_{k} \mid y_{1: k-1}\right)=\int p\left(x_{k} \mid x_{k-1}\right) p\left(x_{k-1} \mid y_{1: k-1}\right) d x_{k-1}
$$

where $p\left(x_{k} \mid x_{k-1}\right)$ is the transition probability of the state define by Eq. (6), $p\left(x_{k-1} \mid y_{1: k-1}\right)$ is the marginal distribution defined by Eq. (7), and $p\left(x_{k} \mid y_{1: k-1}\right)$ is the prior distribution. When a new measurement $y_{k}$ becomes available, the filtering step is executed to calculate the posterior pdf $p\left(x_{k} \mid y_{1: k}\right)$ at time instant $k$. The filtering step uses the new observation $y_{k}$, prior pdf from prediction step $p\left(x_{k} \mid y_{1: k-1}\right)$, and the likelihood function $p\left(y_{1: k} \mid x_{k}\right)$. The calculation is as follows:

$p\left(x_{k} \mid y_{1: k}\right)=\frac{p\left(y_{1: k} \mid x_{k}\right) p\left(x_{k}\right)}{p\left(y_{1: k}\right)}=\frac{p\left(y_{k}, y_{1: k-1} \mid x_{k}\right) p\left(x_{k}\right)}{p\left(y_{k}, y_{1: k-1}\right)}$

where

$$
p\left(y_{k}, y_{1: k-1}\right)=p\left(y_{k} \mid y_{1: k-1}\right) p\left(y_{1: k-1} \mid x_{k}\right)
$$

$$
p\left(y_{k}, y_{1: k-1} \mid x_{k}\right)=p\left(y_{k} \mid y_{1: k-1}, x_{k}\right) p\left(y_{1: k-1} \mid x_{k}\right)
$$

$$
p\left(y_{1: k-1} \mid x_{k}\right)=\frac{p\left(x_{k} \mid y_{1: k-1}\right) p\left(y_{1: k-1}\right)}{p\left(x_{k}\right)}
$$

Then, the posterior can be written as

$$
p\left(x_{k} \mid y_{1: k}\right)=\frac{p\left(y_{k} \mid y_{1: k-1}, x_{k}\right) p\left(x_{k} \mid y_{1: k-1}\right) p\left(y_{1: k-1}\right) p\left(x_{k}\right)}{p\left(y_{k} \mid y_{1: k-1}\right) p\left(y_{1: k-1}\right) p\left(x_{k}\right)}
$$

Assume that all the observations are independent to each other, we have $p\left(y_{k} \mid y_{1: k-1}, x_{k}\right)=p\left(y_{k} \mid x_{k}\right)$. Then, the posterior possibility distribution can be obtained as:

$$
p\left(x_{k} \mid y_{1: k}\right)=\frac{p\left(y_{k} \mid x_{k}\right) p\left(x_{k} \mid y_{1: k-1}\right)}{p\left(y_{k} \mid y_{1: k-1}\right)}
$$


Equation (14) represents a theoretical solution for state estimation. However, for most non-linear and/or non-Gaussian cases, the analytical solution does not exist. For this reason, a Sequential Monte Carlo (SMC) method, also known as particle filtering, was developed to deal with this problem. Particl filtering provides an approximation to the optimal solution given by Eq. (14) (Orchard \& Vachtsevanos, 2007).

Particle filter uses a set of particles $x_{0: k-1}^{i}, i=0,1, \ldots N-1$ with associated weights $w_{k}^{i}, i=0,1, \ldots N-1$ to represent a non-Gaussian distribution. Each particle can be regarded as a sample in the state space. Instead of studying the propagation of the whole distribution, particle filtering studies the propagation of each particle. The prior and posterior distributions are then estimated by the whole set of particles from prediction step and filtering step, respectively. With the set of particles, the empirical distribution can be obtained as:

$$
\pi_{k}\left(x_{0: k}\right)=\sum_{i=1}^{N} w_{k}^{i} \delta\left(x_{0: k}-\bar{x}_{k}^{i}\right)
$$

where $\bar{x}_{k}^{i}$ represents the location of $N$ particles, $\delta(\cdot)$ denotes the Dirac-delta function. The weights can be determined by the importance sampling principle in many applications, the most basic sequential Importance Sampling Resampling particle filter algorithm or bootstrap filter is sufficient for its simplicity. In this algorithm, importance density function is simply chosen to be equal to the prior possibility distribution. Therefore, the weights can be calculated by

$$
w_{k}^{i}=w_{k-1}^{i} p\left(y_{k} \mid x_{k}^{i}\right)
$$

Finally, $x_{k}$ is obtained by weighted sum of the particles:

$$
\hat{x}_{k}=\sum_{i=1}^{N} w_{k}^{i} x_{k}^{i}
$$

\section{Methodology DeVelopment}

This section will describe the framework of the proposed FDP method, which starts from the particle filter based fault diagnosis and prognosis method and is then followed by the integration of DBN in particle filter. Fig. 3 illustrates the framework of the proposed FDP method.

\subsection{Particle Filter based Diagnosis and Prognosis}

The fault diagnostic algorithm is executed at every time instant $\left\{t_{1}, t_{2}, t_{3}, \cdots, t_{k}, \cdots\right\}$. Assume that there exist a set of particles $\left\{w_{k-1}^{(i)}, x_{0: k-1}^{(i)}\right\}$, where the superscript $i$ denotes the $N$ particles located at $x_{0: k-1}^{(i)}$ with weights being $w_{k-1}^{(i)}$. As mentioned in Section 2, particle filtering algorithm aims to approximate a desired distribution using these particles.

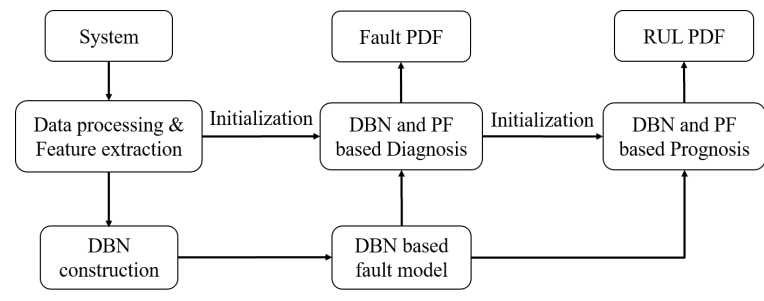

Figure 3. The framework of proposed FDP method

Firstly, $N$ particles with equal weights are taken as initial conditions. Taking these particles in the trained DBN fault dynamic model, a set of new particles can be generated to represent the prior fault pdf. When the measurement becomes available, it is used in the filtering step to update the weight, which is described as:

$$
\begin{gathered}
\omega\left(\bar{x}_{0: k}^{(i)}\right)=\omega_{k-1}^{i} h_{k}\left(y_{1: k} \mid \bar{x}_{0: k}^{i}\right) \\
\omega_{k}^{(i)}=\frac{\omega\left(\bar{x}_{0: k}^{(i)}\right)}{\sum_{i=1}^{N} \omega\left(\bar{x}_{0: k}^{(i)}\right)}
\end{gathered}
$$

Prognosis is the procedure of long-term (multi-step) prediction, that is conducted to depict the degradation of fault state. The ultimate goal of prognosis is to estimate the RUL of the system. The process projects the current fault state pdf, which is from diagnosis and is used as the initial condition of prognosis, into future time instants by the fault state dynamic model. It involves two stages of calculation. The first stage is to calculate the fault state distribution at each future time instant by using the fault state model repeatedly. Since no measurement is available in this long-term prediction, the uncertainty will increase as the prediction horizon increases, which needs to be properly addressed. The second stage is to compare the fault state pdf at each instant with a predefined failure threshold by using the law of total probabilities to get the time to failure (TTF) or RUL distribution. The prognosis is conducted at every time instant to get a RUL distribution. It is noticed that due to the repeated computation of fault state at each future time instants, the prognosis requires significant computing resources and this is one of the main reasons that hinder its online real-time implementation.

In this proposed framework of prognosis, the prediction step is carried out with a fixed time internal from the current time $t_{k}$ to the failure time instant $t_{f}$ when fault state reaches the failure threshold $F_{f}$. The prediction steps are $\left\{t_{k}, t_{k+1}, \cdots, t_{f-1}, t_{f}\right\}$ and the predicted fault state mean value of the distribution at these time instants can be denoted as $\left\{F\left(t_{k}\right), F\left(t_{k+1}\right), \cdots, F\left(t_{f}\right)\right]$. Then, the RUL can be calculated by comparing the distributions of fault state at all prediction steps with the failure threshold. 


\subsection{Integration of DBN in Particle Filter}

The integration of DBN model training process and the particle filtering based FDP is illusrtated in Fig. 4. The major functions shown in Fig. 4 are discussed as follows. The fault dynamic process is described as follows:

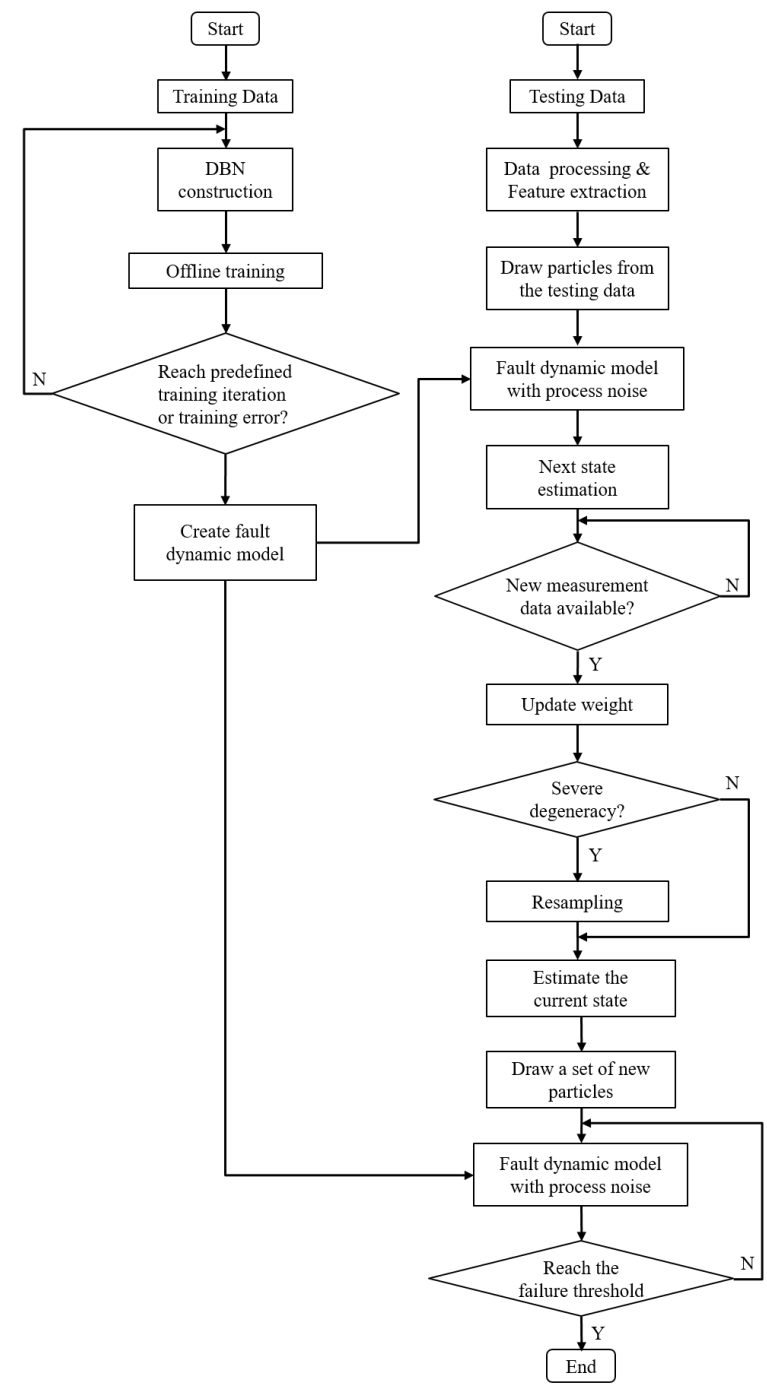

Figure 4. The flowchart of the proposed DNB-based diagnosis and prognosis.

$$
\begin{gathered}
x_{k}=\hat{x}_{k}+\omega_{k} \\
\hat{x}_{k}=g_{k}\left(x_{k-1}, x_{k-2}, x_{k-3}, \cdots, x_{k-m-1}, x_{k-m}\right)
\end{gathered}
$$

where $g_{k}(\cdot)$ is a nonlinear function trained with the DBN, $m$ represents the input vector size of the trained DBN model, $\left\{x_{k-1},, \cdots, x_{k-m}\right\}$ are the previous $m$ states that are used as the input of the DBN model, $\hat{x}_{k}$ represents the output of the DBN model, $\omega_{k}$ represents the process noise, and $x_{k}$ is the predicted state at next time instant with noise.

As shown in Fig. 4, the DBN model involves offline training and online prediction. The detailed algorithm steps can be illustrated as follows.

Step 1: The DBN is trained using the available data to model the fault dynamic process.

Step 2: The fault dynamic model (20) is used in particle filtering algorithm to draw a set of particles. Take the current particles as the input, the prediction of next time instant state can be conducted with the fault dynamic model.

Step 3: Prognosis algorithm is executed repeatedly following the Step 2. This is a multistep prediction that relies mainly on the fault dynamic model denoted by DBN model. The recursively calculation is illustrated as follows:

$$
\left\{\begin{array}{l}
x_{k}^{i}=g\left(x_{k-1}^{i}, x_{k-2}^{i}, x_{k-3}^{i}, \cdots, x_{k-m}^{i}\right)+\omega_{k-1} \\
x_{k+1}^{i}=g\left(x_{k}^{i}, x_{k-1}^{i}, x_{k-2}^{i}, \cdots, x_{k-m-1}^{i}\right)+\omega_{k} \\
\quad \vdots \\
x_{k+n}^{i}=g\left(x_{k+n-1}^{i}, x_{k+n-2}^{i}, \cdots, x_{k+n-m}^{i}\right)+\omega_{k+n-1}
\end{array}\right.
$$

where $g(\cdot)$ represents the fault dynamic model obtained by DNB training, $\omega$ is the processing noise. It is executed recursively until the predicted fault state reaches the defined failure threshold.

In the diagnosis step, after the prediction is obtained via DBN, the new measurement comes in. The weights are then updated according to (18) and (19). If there exists severe degeneracy, which is indicated by the increase of weight variances such that all but one particle have trivial weights, the particles are resampled to solve the problem.

Step 4: Repeat Step 2 and Step 3 until the diagnosis and prognosis process is finished.

\section{EXPERimental Results}

In this section, the proposed DBN based FDP approach will be demonstrated with a particle filtering algorithm in a lithium-ion battery capacity degradation case study. The experiments were carried out for lithium-ion batteries with a rated capacity of $1.1 \mathrm{Ah}$. Arbin BT2000 system was utilized to conduct the charge and discharge of the battery. Fig. 5 shows the capacity degradation curves where the failure threshold is set as $0.35 \mathrm{Ah}$. In other words, the battery is considered as failure when the capacity degrades to the defined value.

\subsection{Fault Dynamic Model}

In this work, the deep belief networks are trained to depict the battery capacity degradation curves. The main parameters of the DBNs are shown in Table 1. In the training process, samples are divided into training data sets and testing data sets. In this work, two battery dataset CS2_35 and CS2_36 


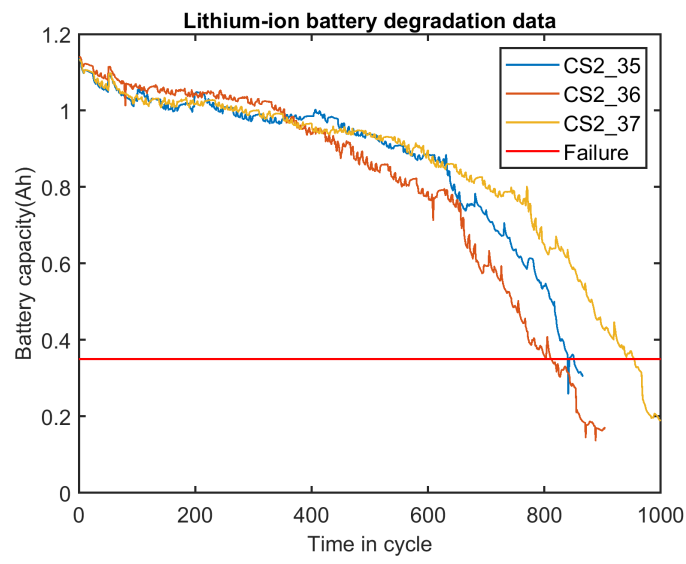

Figure 5. The Li-ion battery degradation data.

are used to train the DBN model. The training input-output data pairs are described as follows:

$$
\left\{\begin{array}{c}
\left\{x_{1}, x_{2}, \ldots, x_{119}, x_{120}\right\} \rightarrow \bar{x}_{121} \\
\left\{x_{2}, x_{3}, \ldots, x_{120}, x_{121}\right\} \rightarrow \bar{x}_{122} \\
\left\{x_{3}, x_{4}, \ldots, x_{121}, x_{122}\right\} \rightarrow \bar{x}_{123} \\
\vdots \\
\vdots
\end{array}\right.
$$

Table 1. Training parameters of DBN model

\begin{tabular}{cc}
\hline \hline Parameters & Value \\
\hline \hline The unit number of input layer & 120 \\
The number of RBM & 2 \\
The unit number of hidden layer & 30 \\
The unit number of output layer & 20 \\
Learning rate of RBM & 0.1 \\
Initial momentum of RBM & 0.5 \\
Iterations of each RBM & 500 \\
Iteration of conjugate gradient & 0.5 \\
\hline
\end{tabular}

The DBN model is then trained based on the above inputoutput data set. To evaluate the performance of the trained DBN model, a third battery dataset CS2_37 is used to test the trained model. Fig. 6 shows the training results, which demonstrates the performance of one step prediction.

In prognosis, however, it is of interest to assess whether the trained DBN model can sufficiently and accurately depict the battery capacity degradation. To test the performance of long time prediction, the following testing process is conducted:

$$
\left\{\begin{array}{cc}
\left\{x_{1}, x_{2}, \ldots, x_{119}, x_{120}\right\} & \rightarrow \bar{x}_{121} \\
\left\{x_{2}, x_{3}, \ldots, x_{120}, \bar{x}_{121}\right\} & \rightarrow \bar{x}_{122} \\
\left\{x_{3}, x_{4}, \ldots, \bar{x}_{121}, \bar{x}_{122}\right\} & \rightarrow \bar{x}_{123} \\
\vdots & \vdots
\end{array}\right.
$$

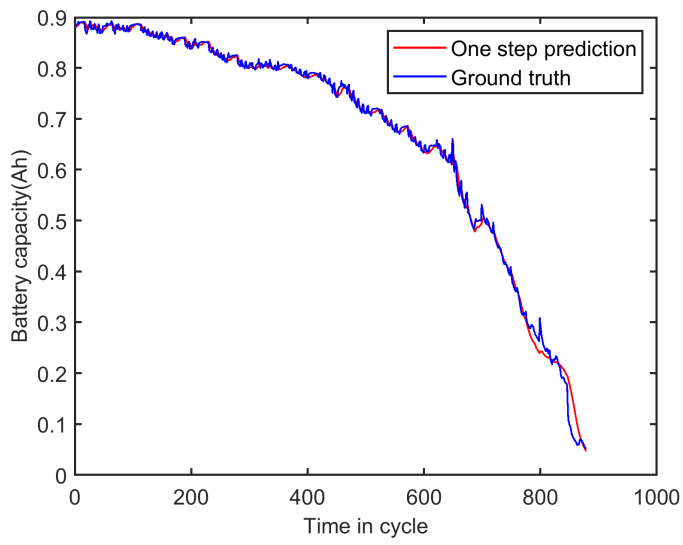

Figure 6. One step prediction result of trained DBN model.

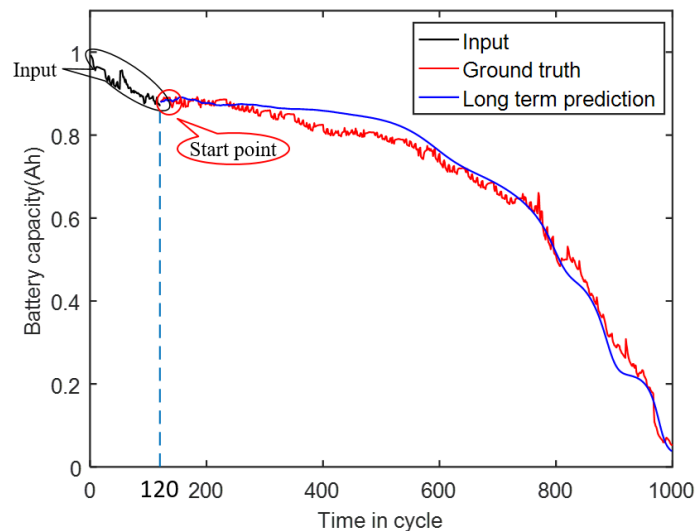

Figure 7. Long-term prediction of trained DBN model.

Fig. 7 shows the result of long time prediction started from the 120-th data point. It is clear that the trained DBN model can accurately depict the battery capacity degradation. In order to give an quantitative evaluation of the prediction performance, root-mean-square (RMS) error defined in Eq. (25) is used. For the results shown in Fig. 7, the RMS error of the prediction is 0.0133 , which indicates the superior performance of prediction accuracy.

$$
\text { rmse }=\sqrt{\frac{\sum_{i=1}^{M}\left(y_{i}-\hat{y}_{i}\right)^{2}}{M}}
$$




\subsection{FDP Results}

For battery degradation, since the state and life are not observable, diagnostic and prognostic algorithm is used to estimate the states, such as state of health ( $\mathrm{SOH})$, state of charge (SOC), and RUL. In this section, the proposed approach is tested with the battery $\mathrm{SOH}$ degradation with chargedischarge cycles.

\subsubsection{DBN based diagnosis and prognosis}

In the proposed FDP method, the diagnosis and prognosis model is the fault dynamic model trained with DBN. In the diagnosis, a particle filter with 500 particles is used. The unit number of the input layer is 120 . Fig. 8 shows the diagnostic results at the 500th cycle. Note that since the input size is 120 , the diagnosis is implemented from the 121st data and the first 120 data are not shown in this figure. The upper subfigure shows battery capacity estimation (given by magenta) compared with the measurements from the battery test system (given by blue). The lower subfigure shows a comparison of the initial baseline pdf (obtained from the battery before degradation and given by green) against the real time estimation pdf (from the proposed method and given by magenta) at the 500th cycle.
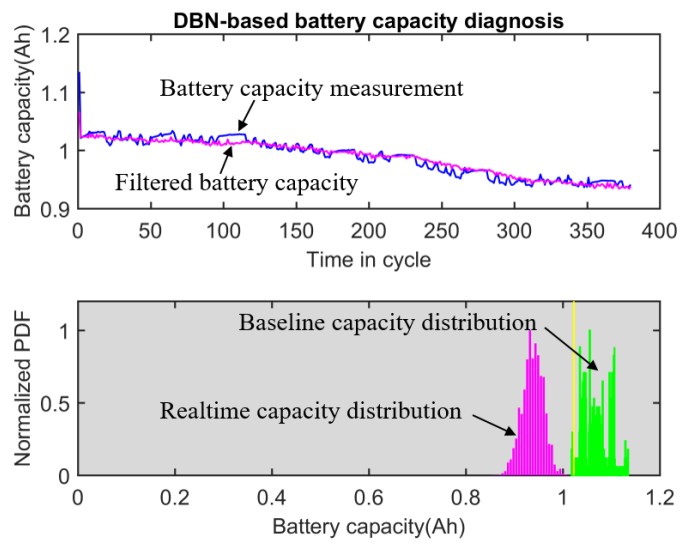

Figure 8. Experimental result of DBN based diagnosis. Upper subfigure shows the comparison of estimated mean capacity (given by magenta) against the measurement (given by blue). Lower subfigure shows the comparison of baseline pdf (given by green) against the real-time estimation pdf (given by magenta).

Prognosis is then conducted to predict the future battery capacity state and estimate the RUL. Prognosis predicts future battery capacity degradation recursively until it reaches the defined failure threshold. Since prognosis is time consuming, 20 particles are used in prognosis to reduce the computation time. Fig. 9 shows the prognostic results at the 500th cycle, which shows the mean value, $95 \%$ confidence interval of the RUL pdf, and the estimated RUL pdf. The figure shows that the predicted failure time is at the 852th cycle, and the RUL is 352 cycles. The $95 \%$ confidence interval of the RUL pdf is $[345,366]$.

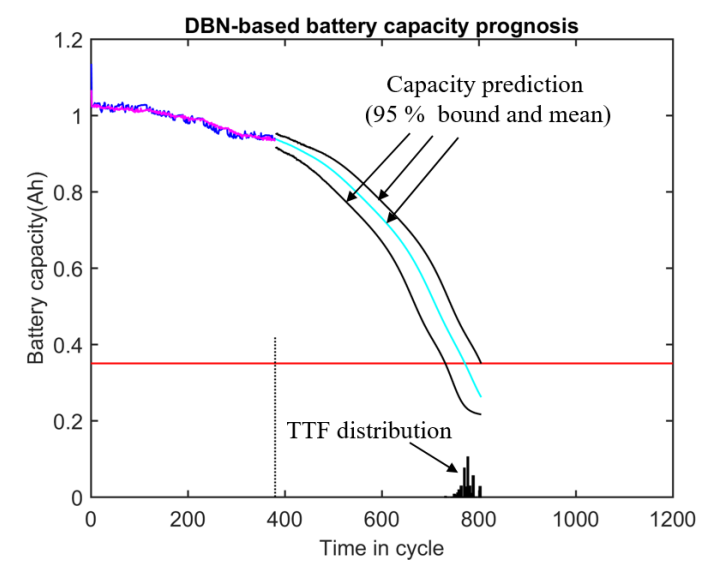

Figure 9. Experimental result of DBN based prognosis.

This work adopts $\alpha-\lambda$ metrics (Saxena, Celaya, Saha, Saha, \& Goebel, 2010), which determines whether the prediction at any particular time instance falls within a specified precision range. Fig. 13 shows the $\alpha-\lambda$ metrics of the proposed method with $\alpha=0.3$. It is clear from this figure that the predicted RUL at all the time instants always fall inside the defined $\alpha$ bounds around the true RUL.

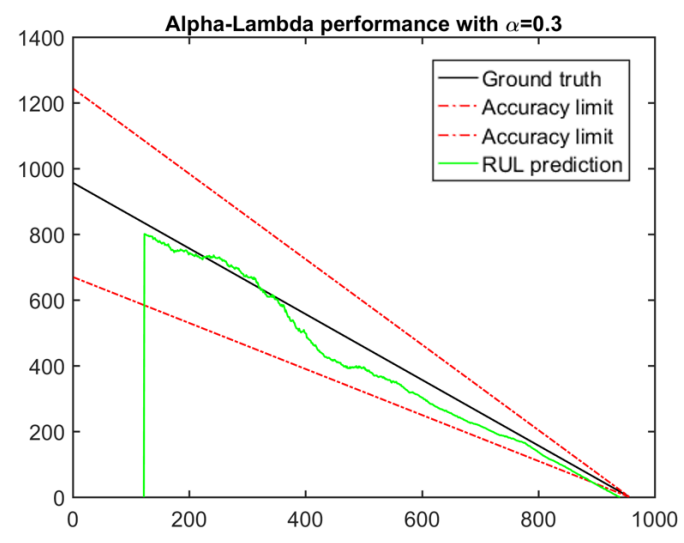

Figure 10. Experimental result of DBN based RUL.

\subsubsection{Particle filter based diagnosis and prognosis}

In order to demonstrate the effectiveness of the proposed FDP method, it is compared with traditional particle filtering approach (B. Zhang et al., 2011). In the traditional particle filtering FDP, the fault dynamic model is given by

$$
C(t+1)=C(t)-\beta\left|p_{1}\left(p_{2}+p_{3} t+p_{4} t^{2}\right)\right|^{p_{5}}+\omega(t)
$$

where $C$ is the battery capacity, $t$ is the time given by charge-discharge cycle, $p_{1} \sim p_{5}$ are the parameters of the model given by $p=\left[5 e^{-5},-215,4.8,-0.0135,0.4\right], \beta \sim$ 
$N\left(3.8 e^{-3}, 5 e^{-5}\right)$ is a hyper parameter, and $\omega$ is the model noise.

To make the comparison fair, traditional particle filter with 500 particles and 20 particles are used in diagnosis and prognosis respectively. Other parameters of the particles filter are same with the parameters used in the proposed DBN-based particle filter. Figs. 11 and 12 show the diagnosis and prognosis results at the 500th cycle from traditional particle filter approach. Fig. 13 shows the RUL prediction in terms of $\alpha-\lambda$ metrics. It is clear that some of the RUL predictions fall out of the $\alpha$ bounds of the true RUL, which indicates low accuracy in prognosis.
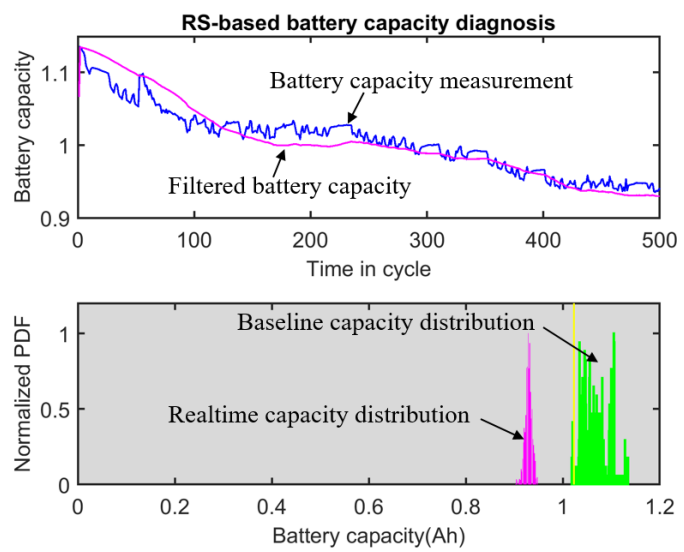

Figure 11. Experimental result of particle filter based diagnosis. Upper subfigure shows the comparison of estimated mean capacity (given by magenta) against the measurement (given by blue). Lower subfigure shows the comparison of baseline pdf (given by green) against the real-time estimation pdf (given by magenta).

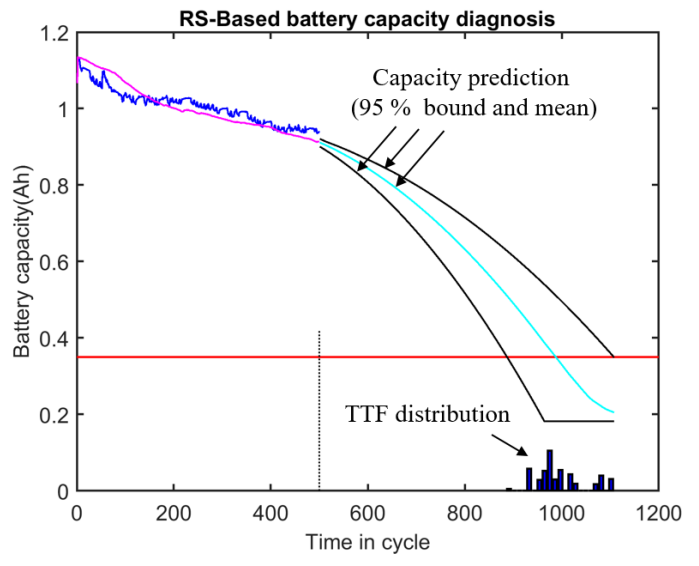

Figure 12. Experimental result of particle filter based prognosis.

The comparison of the DBN based FDP and traditional model based FDP in Figs. 8 and 11 shows that these two approaches show comparable performance in diagnosis. The proposed

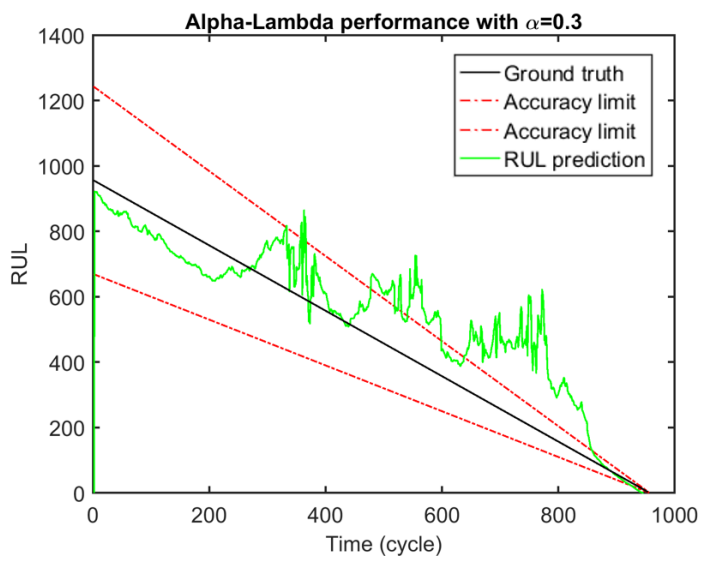

Figure 13. Experimental result of model based RUL.

DBN-based approach has a wider distribution of fault state estimation. In terms of prognosis, it is clear from Figs. 10 and 13 that the proposed DBN-based FDP approach has better performance in accuracy and precision than the traditional model-based FDP approach. Moreover, the RUL prediction of traditional model based FDP has very large fluctuation, which indicates the inconsistency of prediction. On the contrary, the proposed DBN-based FDP approach has very stable prediction performance, which greatly benefits the decisionmaking. In summary, the comparison shows that the DBNbased FDP approach has better performance than traditional model-based FDP approach.

\section{Conclusion}

This paper proposes a DBN-based FDP approach that integrates with particle filtering. The theoretical background of DNB and particle filtering are discussed in details, along with the training of DNB fault dynamic model. In the proposed approach, the constructed DBN model is trained offline using the available historical data. The trained DBN model is then integrated into the particle filtering algorithm for FDP. In order to evaluate the performance of the proposed approach, a case study of lithium-ion battery capacity degradation was presented and compared with the traditional particle filtering FDP approach. The results show great performance improvement from the proposed approach. It is worth mentioning that the proposed DNB-based approach can be integrated with other algorithms such as Kalman filter, Extended Kalman filter, support vector machine, etc. From this prospective, the proposed DBN-based approach is a generic solution that can be applied to a variety of systems. Our future work will focus on introducing uncertainty management and improving the training efficiency of the proposed approach . 


\section{ACKNOWLEDGMENT}

The work is partially supported by the ASPIRE grant program at the University of South Carolina.

\section{REFERENCES}

Bengio, Y., Lamblin, P., Popovici, D., \& Larochelle, H. (2007). Greedy layer-wise training of deep networks. In Advances in neural information processing systems (pp. 153-160).

Biswas, S. S., Srivastava, A. K., \& Whitehead, D. (2015). A real-time data-driven algorithm for health diagnosis and prognosis of a circuit breaker trip assembly. IEEE Transactions on Industrial Electronics, 62(6), 38223831.

Chen, Z., \& Li, W. (2017). Multisensor feature fusion for bearing fault diagnosis using sparse autoencoder and deep belief network. IEEE Transactions on Instrumentation and Measurement, 66(7), 1693-1702.

Hu, S., Wang, L., Mao, J., Gao, C., Zhang, B., \& Yang, S. (2018). Synchronous online diagnosis of multiple cable intermittent faults based on chaotic spread spectrum sequence. IEEE Transactions on Industrial Electronics.

Jardine, A. K., Lin, D., \& Banjevic, D. (2006). A review on machinery diagnostics and prognostics implementing condition-based maintenance. Mechanical Systems and Signal Processing, 20(7), 1483-1510.

Kan, M. S., Tan, A. C., \& Mathew, J. (2015). A review on prognostic techniques for non-stationary and nonlinear rotating systems. Mechanical Systems and Signal Processing, 62, 1-20.

Kang, S., \& Meng, H. (2014). Statistical parametric speech synthesis using weighted multi-distribution deep belief network. In Fifteenth annual conference of the international speech communication association.

Mosallam, A., Medjaher, K., \& Zerhouni, N. (2016). Data-driven prognostic method based on bayesian approaches for direct remaining useful life prediction. Journal of Intelligent Manufacturing, 27(5), 10371048 .

Oquab, M., Bottou, L., Laptev, I., \& Sivic, J. (2014). Learning and transferring mid-level image representations using convolutional neural networks. In Computer vision and pattern recognition (cvpr), 2014 ieee conference on (pp. 1717-1724).

Orchard, M. E., \& Vachtsevanos, G. J. (2007). A particle filtering-based framework for real-time fault diagnosis and failure prognosis in a turbine engine. In Control \& automation, 2007. med'07. mediterranean conference on (pp. 1-6).

Orchard, M. E., \& Vachtsevanos, G. J. (2009). A particlefiltering approach for on-line fault diagnosis and failure prognosis. Transactions of the Institute of Measurement and Control, 31(3-4), 221-246.

Saxena, A., Celaya, J., Saha, B., Saha, S., \& Goebel, K. (2010). Metrics for offline evaluation of prognostic performance. International Journal of Prognostics and Health Management, 1(1), 4-23.

Tamilselvan, P., \& Wang, P. (2013). Failure diagnosis using deep belief learning based health state classification. Reliability Engineering \& System Safety, 115, 124-135.

Titos, M., Bueno, A., García, L., \& Benítez, C. (2018). A deep neural networks approach to automatic recognition systems for volcano-seismic events. IEEE Journal of Selected Topics in Applied Earth Observations and Remote Sensing.

Van Tung Tran, F. A., \& Ball, A. (2014). An approach to fault diagnosis of reciprocating compressor valves using teager-kaiser energy operator and deep belief networks. Expert Systems with Applications, 41(9), 41134122.

Wang, X., Li, Y., Rui, T., Zhu, H., \& Fei, J. (2015). 1593. bearing fault diagnosis method based on hilbert envelope spectrum and deep belief network. Journal of $\mathrm{Vi}$ broengineering, 17(3).

Wang, Y., Peng, Y., Zi, Y., Jin, X., \& Tsui, K.-L. (2016). A two-stage data-driven-based prognostic approach for bearing degradation problem. IEEE Transactions on Industrial Informatics, 12(3), 924-932.

Yan, W., Zhang, B., Wang, X., Dou, W., \& Wang, J. (2016). Lebesgue-sampling-based diagnosis and prognosis for lithium-ion batteries. IEEE Transactions on Industrial Electronics, 63(3), 1804-1812.

Yan, W., Zhang, B., Zhao, G., Tang, S., Niu, G., \& Wang, $X$. (2018). Battery management system with lebesgue sampling-based extended kalman filter. IEEE Transactions on Industrial Electronics.

Zhang, B., Sconyers, C., Byington, C., Patrick, R., Orchard, M. E., \& Vachtsevanos, G. (2011). A probabilistic fault detection approach: Application to bearing fault detection. IEEE Transactions on Industrial Electronics, 58(5).

Zhang, C., Lim, P., Qin, A., \& Tan, K. C. (2017). Multiobjective deep belief networks ensemble for remaining useful life estimation in prognostics. IEEE Transactions on Neural Networks and Learning Systems, 28(10), 2306-2318.

Zhang, Z., Wang, Y., \& Wang, K. (2013). Fault diagnosis and prognosis using wavelet packet decomposition, fourier transform and artificial neural network. Journal of Intelligent Manufacturing, 24(6), 1213-1227.

Zhao, G., Liu, X., Zhang, B., Liu, Y., Niu, G., \& Hu, C. (2018). A novel approach for analog circuit fault diagnosis based on deep belief network. Measurement, $121,170-178$. 
Zhao, G., Liu, X., Zhang, B., Zhang, G., Niu, G., \& Hu, C. (n.d.). Bearing health condition prediction using deep belief network.

Zhao, R., Wang, D., Yan, R., Mao, K., Shen, F., \& Wang, J. (2018). Machine health monitoring using local featurebased gated recurrent unit networks. IEEE Transactions on Industrial Electronics, 65(2), 1539-1548.

\section{BIOGRAPHIES}

Guangxing Niu received the B.A. degree from North University of China, Taiyuan, China, in 2014, and the M.S.E. degree from Harbin Institute of Technology, Harbin, China, in 2016, both in electrical engineering. He is currently working toward the Ph.D. degree in electrical engineering from the University of South Carolina, Columbia, SC, USA. His current research focuses on the data-driven based algorithms of diagnosis and prognosis for lithium ion batteries and industrial systems.

Shijie Tang received the B.E. degree in Electrical Engineering from Yanshan University, Qinhuangdao, China, in 2016. $\mathrm{He}$ is currently pursuing the Ph.D. degree in Electrical Engineering at University of South Carolina, Columbia, South Carolina, US. His current research interests are Fault Diagnosis, Prognostic and Health Management, Machine Learning and Control Theory.

Zhichao Liu received his B.E and M.E. degrees in electrical engineering from East China University of Science and
Technology, China, in 2008 and 2011, respectively. He is currently working towards the $\mathrm{Ph} . \mathrm{D}$. degree in electrical engineering, University of South Carolina, Columbia, SC, USA. From 2008 to 2011, he was with Shanghai Baosight software, Co., Ltd. Shanghai, China. His research interest includes signal processing, aging cable diagnosis, advanced control theory and applications, digital control of power converter, and power electronics.

Guangquan Zhao received the B.E., M.E., and Ph.D. degrees in instrumentation science and technology from Harbin Institute of Technology, Harbin, China, in 2000, 2002, and 2007, respectively. In 2002, he joined the Department of Automatic Test and Control, Harbin Institute of Technology, where he has developed his research activity and is currently an Associate Professor. His research interests include deep learning, data-driven fault diagnosis and prognosis, and their applications to various engineering systems.

Bin Zhang received the B.E. and M.E. degrees from Nanjing University of Science and Technology, Nanjing, China, and the Ph.D. degree from Nanyang Technological University, Singapore, in 2007. Before he joined the Department of Electrical Engineering, University of South Carolina, Columbia, SC, USA, he was with General Motors R\&D, Detroit, MI; Impact Technologies, Rochester, NY; and Georgia Institute of Technology, Atlanta, GA, USA. He has published over 100 technical papers. His current research interests include prognostics and health management, intelligent systems and controls, and their applications to various engineering systems. 